\title{
Silent threat? A retrospective study of screening practices for pulmonary arteriovenous malformations in patients with hereditary haemorrhagic telangiectasia*
}

\author{
Misha M. Verkerk' , Claire L. Shovlin², Valerie J. Lund \\ National Heart and Lung Institute, Imperial College, London, United Kingdom \\ Professorial Unit, Royal National Throat Nose and Ear Hospital, London, United Kingdom
}

Rhinology 50: 277-283, 2012

DOI:10.4193/Rhino12.043

*Received for publication:

March 10, 2012

Accepted: April 5, 2012

\section{Summary}

Background: Patients with hereditary haemorrhagic telangiectasia (HHT) are at risk of pulmonary arteriovenous malformations (PAVMs) that may be complicated by stroke and brain abscess. ENT surgeons are well placed to direct patients to screening, which was recommended for all HHT patients in recently published international guidelines.

Methodology/Principal: A retrospective study of patients with known HHT was performed based on responses to a telephone questionnaire. Epistaxis was assessed using a validated epistaxis severity scoring system.

Results: 123 patients responded, with ages ranging from 14-86 years. 80\% of patients experienced their first symptom of HHT by 30 years old. Epistaxis was assessed at time of questionnaire as mild, moderate or severe. 71 patients underwent screening for PAVMs. 30 patients reported PAVMs detected by screening. 18 patients received treatment and 12 patients were found to have PAVMs too small for treatment. The modal screening method was CT and 15 patients with treatable PAVMs received trans-catheter embolisation. Only 9 patients reported being under long term follow up for PAVMs. Shortness of breath and migraine were more common amongst patients found to have PAVMs than those without PAVMs. There was no difference in age of onset of HHT symptoms or epistaxis severity between patients with PAVMs and those without.

Conclusions: PAVMs are common in HHT patients and carry a risk of morbidity and mortality. Safe and effective treatment exists for PAVMs although a significant minority of patients has received no screening to date. Clinicians should refer all patients for screening regardless of symptoms.

Key words: hereditary hemorrhagic telangiectasia, arteriovenous malformations, embolization, therapeutic, epistaxis, migraine, shortness of breath

\section{Introduction}

Hereditary haemorrhagic telangiectasia (HHT, Osler-WeberRendu syndrome) is an autosomal dominant vascular disorder with an incidence of 1 in $5-8,000{ }^{(1)}$. Originally described in the 19th century, the disease is characterized by spontaneous and recurrent epistaxis, telangiectases in characteristic sites (nose, lips, mouth, fingers) and arteriovenous malformations in internal organs including lung, brain, liver and GI tract ${ }^{(3)}$. ENT surgeons play an important role in the management of patients with $\mathrm{HHT}$, as spontaneous recurrent nosebleeds occur in around $90 \%$ of patients, often commencing before school age ${ }^{(4)}$. However, whilst most patients do not suffer life-limiting consequences of $\mathrm{HHT}$, it is the arteriovenous malformations (AVMs) in pulmonary, cerebral and hepatic circulations that carry the greatest risk of mortality and are frequently 'silent' or asymptomatic ${ }^{(1,5-8)}$. Recent data suggests that asymptomatic pulmonary arteriovenous malformations (PAVMs) occur in as many as $50 \%$ of patients with $\mathrm{HHT}^{(9)}$. The resulting right-to-left 
shunt may have sequelae ranging from dyspnoea and $\mathrm{mi}-$ graine to massive haemoptysis, stroke/transient ischaemic attack (TIA) and brain abscess ${ }^{(10-13)}$, the latter presumed to occur through paradoxical embolism. PAVMs rarely regress spontaneously and tend to increase in size over time, especially if multiple, which is commonly the case in $\mathrm{HHT}^{(14,15)}$. Mortality rates of untreated symptomatic patients vary in historical reviews from $4-22 \%$ and up to $40 \%$ in severe cases ${ }^{(16)}$.

Low risk detection methods for PAVMs and safe and effective treatment have meant that current international guidelines recommend the routine screening of all HHT patients for pulmonary AVMs after initial assessment ${ }^{(17)}$. The recommended screening methods are trans-thoracic contrast echocardiography (TTCE) followed by confirmatory computerized tomography (CT). The recommended treatment is trans-catheter embolisation, which has been shown to be safe and effective in reducing or abolishing the risk of paradoxical embolic stroke and brain abscess ${ }^{(18)}$. Follow-up of all patients is required every 1-10 years depending on screening outcome ${ }^{(17)}$.

The main objectives of this study were to compare current screening procedures at a major ENT referral hospital with international guidelines by assessing: 1) how many patients with HHT were screened for PAVMs; 2 ) how often PAVMs are detected within this group, and; 3 ) how they are managed. A secondary objective was to assess whether there was a positive correlation between the presence of PAVMs and the age of onset of HHT or epistaxis severity.

\section{Materials and methods}

Patients

Selection criteria for the study were patients who fulfilled the Curaçao criteria for $\mathrm{HHT}^{(3)}$ and who had been treated for epistaxis at the Royal National Throat Nose and Ear (RNTNE) Hospital, London, UK over the last 25 years. 132 patients were asked to complete the questionnaire.

\section{Consent}

All patients gave verbal consent to the use of their anonymised information solely for this study.

\section{Questionnaire}

Patients were invited to answer a confidential questionnaire over the telephone. Epistaxis severity was formally graded on a scale of 0 - 10 using six weighted questions in an Epistaxis Severity Score questionnaire designed for use in patients with $\mathrm{HHT}{ }^{(19)}$. This included questions on frequency, duration and intensity of nose-bleeds over the previous month, whether the patient had ever received medical attention for nose-bleeds, whether they were currently anaemic and whether they had ever received a blood transfusion specifically for nose-bleeds. Scores were then categorized as mild (0 - 4), moderate (4 - 7) and severe (7 - 10).

Further questions included age of onset of HHT symptoms, details of treatments received for nose-bleeds, the presence of symptoms of pulmonary AVMs (shortness of breath on exertion, chest pain, coughing up blood, migraine) over the previous month (or before treatment for PAVMs if appropriate) and whether they had ever had a stroke/transient ischaemic attack (TIA) or brain abscess. The questionnaire also included the results and outcome of any screening investigations or treatments for PAVMs. For the purposes of this study, patients were not asked any questions related to arteriovenous malformations in other organs.

\section{Analysis}

Data is presented as raw data, percentages and mean \pm standard error of the mean where appropriate. Epistaxis severity scores were compared statistically using the MannWhitney U Test (SPSS Inc., Chicago, IL, USA) and symptoms were compared using Fisher's Exact Test (Graphpad QuickCalcs website: http://www.graphpad.com/quickcalcs/contingency2. cfm, accessed March 2012).

\section{Results}

\section{Patient demographics}

We received 123 full responses to the questionnaire, with 5 patients declining to respond and 4 patients who were excluded because they had started but not completed a screening process. There were 58 male patients and 65 female patients. At onset of first symptoms, the average age of respondents ranged from $2-70$ years (mean $19.1 \pm 1.2$ years). $80 \%$ of patients had experienced their first symptoms by the age of 30 (see Figure 1). At the time of the questionnaire, age of respondents ranged from 14 - 86 years (mean $57.0 \pm 1.8$ years).

\section{HHT symptoms}

One hundred fourteen patients (92.7\%) described their first symptom as nose-bleeding. However, bleeding from other sites, including the skin, mouth, in stool and from the eyes was also reported (Table 1). 106 patients (86.2\%) felt subjectively that their nose-bleeds had got worse with age. Epistaxis severity in the last month was assessed using the normalized Epistaxis Severity Score. Patients were classified as experiencing mild (26 patients, $21.1 \%$ ), moderate (52 patients, $42.2 \%$ ) or severe epistaxis (45 patients, $36.6 \%$, Figure 2). 73 patients $(59.3 \%)$ reported taking regular iron supplementation for anaemia.

\section{HHT treatment}

Treatments reported for HHT-related epistaxis included laser, septodermoplasty, nasal closure (Young's procedure), cautery, tranexamic acid, sponge packs, oestrogen cream and Vaseline (Table 2). Laser was the most frequently reported single treat- 
Table 1. HHT symptoms.

\begin{tabular}{cc} 
Bleeding site & $\begin{array}{c}\text { Number with symptom } \\
(\% \text { of total, } \mathbf{n}=123)\end{array}$ \\
\hline Nose & $123(100)$ \\
Mouth & $79(64)$ \\
Other skin site e.g. finger, ear & $60(49)$ \\
Stool & $34(28)$ \\
Eyes & $11(9)$
\end{tabular}

Respondents were asked to recall lifetime occurrence of bleeding symptoms. Sum of percentages is greater than $100 \%$ as patients reported multiple symptoms.
Table 2. HHT treatment.

\begin{tabular}{|c|c|}
\hline Treatment & $\begin{array}{c}\text { Number of patients } \\
\text { (\% of total, } \mathbf{n = 1 2 3 )}\end{array}$ \\
\hline Laser & $105(85)$ \\
\hline Septodermoplasty & $50(41)$ \\
\hline Young's procedure (nasal closure) & $26(21)$ \\
\hline Sprays/ointments/creams & $89(72)$ \\
\hline Tranexamic acid & $29(24)$ \\
Cautery & $20(16)$ \\
\hline
\end{tabular}

Sum of percentages is greater than $100 \%$ as patients reported multiple treatment modalities.

Table 3. Symptoms and signs of PAVMs.

\begin{tabular}{|c|c|c|}
\hline & $\begin{array}{l}\text { Patients with PAVMs } \\
\quad(\% \text { total, } n=30)\end{array}$ & $\begin{array}{l}\text { Patients without PAVMs } \\
(\% \text { total, } n=41)\end{array}$ \\
\hline \multicolumn{3}{|l|}{ Respiratory } \\
\hline Shortness of breath on exertion & $21 *(70.0)$ & $17(41.5)$ \\
\hline Chest pain & $11(36.7)$ & $15(36.6)$ \\
\hline Haemoptysis & $4(13.3)$ & $3(7.3)$ \\
\hline \multicolumn{3}{|l|}{ Neurological } \\
\hline Migraine & $13^{*}(43.3)$ & $10(24.4)$ \\
\hline Stroke/TIA & $7(23.3)$ & $3(7.3)$ \\
\hline Brain abscess & $2(6.6)$ & $0(0)$ \\
\hline Asymptomatic & $4(13.3)$ & $13(31.7)$ \\
\hline
\end{tabular}

Patients were asked to recall symptoms in the last month (or before treatment for PAVMs if appropriate). Significant differences $(p<0.05)$ are: ${ }^{*}$, vs No PAVMs (Fisher's Exact Test).

ment (105 patients, $85.4 \%)$, followed by septodermoplasty $(50$ patients), tranexamic acid (29 patients) and nasal closure (26 patients) although 89 patients reported use of an ointment, cream or spray to relieve symptoms.

\section{PAVM symptoms and signs}

The presence of certain cardinal symptoms and signs of pulmonary AVMs was assessed by asking patients to recall symptoms in the last month (or before treatment for PAVMs, if appropriate). Across the sample population, shortness of breath (64 patients), chest pain (37 patients) and migraine (36 patients) were commonly reported, with few patients reporting haemoptysis (6 patients). Eleven patients reported stroke or transient ischaemic attack (TIA) and 3 patients reported brain abscess.

Patients with PAVMs detected by screening were significantly more likely to report shortness of breath or migraine over the previous month than those without PAVMs (Table 3, p $<0.05$, Fisher's Exact Test). There was no difference found between the two groups in relation to haemoptysis, chest pain, stroke/ TIA or brain abscess. The number of patients reporting none of the cardinal symptoms or signs was not significantly different between the group with PAVMs and those without.

\section{PAVM screening}

Seventy-one patients reported undergoing screening for PAVMs and 52 patients reported that they had not been screened. Thirty patients reported detection of PAVMs following screening (42.3\% of the screened population). Patients reported a variety of screening procedures, the most common being computerized tomography (CT, 58 patients). Other methods reported included spirometry (8 patients), trans-thoracic contrast echocardiography (TTCE, 5 patients), plain x-ray (3 patients) and magnetic resonance imaging (MRI, 1 patient). Some patients reported more than one screening modality. 


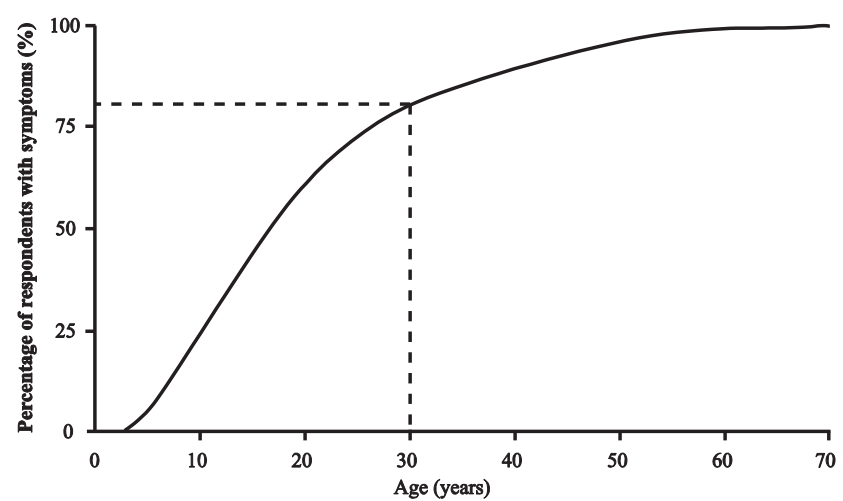

Figure 1. Age of onset of HHT symptoms. Respondents $(n=123)$ were asked at what age they first experienced bleeding related to hereditary haemorrhagic telangiectasia (HHT).

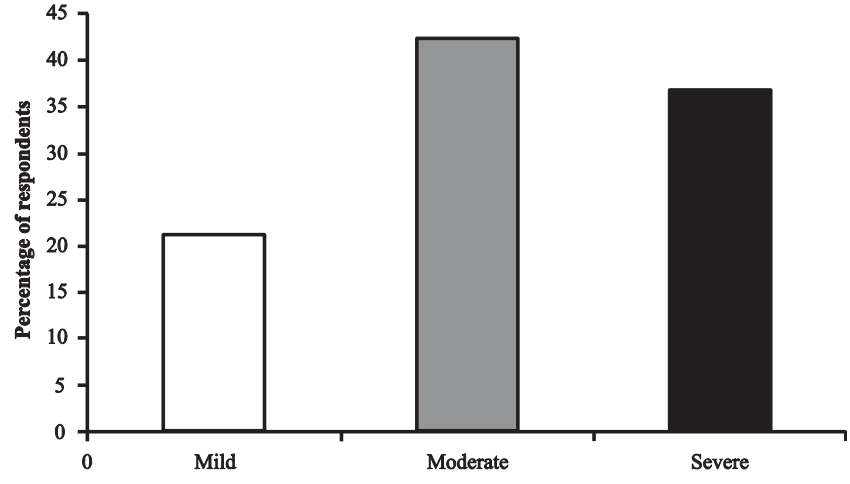

Figure 2. Epistaxis Severity Scores. Respondents $(n=123)$ were categorized with mild, moderate and severe epistaxis as per Hoag et al. ${ }^{(19)}$.

\section{PAVM management}

Of the 30 patients who reported detection of PAVMs, 18 patients reported receiving treatment (Figure 3). Fifteen patients underwent embolisation alone, one had combined surgery and embolisation and two had surgery alone. Twelve patients who were found to have PAVMs reported receiving no treatment. Reasons for no treatment included PAVMs that were too small for embolisation and co-morbidity (one patient was advised against treatment because of co-existing pulmonary hypertension). Nine patients ( $13 \%$ of the screened population) reported being under follow-up for PAVMs.

\section{Epistaxis, age of onset and PAVMs}

To assess whether age of onset of HHT symptoms or epistaxis severity could be used to guide screening decisions, age of onset and severity of epistaxis were compared with the presence of PAVMs. There was no difference in age of onset of HHT symptoms between patients with PAVMs and those without (PAVMs: $16.4 \pm 2.0$ years; No PAVMs: $18.9 \pm 1.9$ years, $p=0.448$, Mann-Whitney U Test). There was no difference in mean epistaxis severity scores between patients with PAVMs and those without (PAVMs: $6.4 \pm 0.37$; No PAVMs: $5.9 \pm 0.35, p=0.300$, Mann-Whitney U Test). In order to assess whether patients with more severe epistaxis were being screened more than those with milder symptoms, severity of epistaxis was compared against screening status. There was no difference in mean epistaxis severity scores between those who attended screening and those who did not (Screened: $6.1 \pm$ 0.25; Not screened: 5.6 \pm 0.29. $p=0.233$, Mann-Whitney U Test).

\section{Discussion}

In light of the risk posed by pulmonary arteriovenous malformations (PAVMs) and recent clinical guidelines recommending screening for PAVMs in all patients with hereditary haemorrhagic telangiectasia $(\mathrm{HHT}){ }^{(17)}$, this clinical study profiled a series of 123 patients with the disease and examined the screening practices of a tertiary ENT referral centre. A full evaluation of
HHT management is beyond the scope of this article, but a recent clinical and scientific review has been published ${ }^{(4)}$. The salient findings of this study were: 1) $80 \%$ of patients reported first symptoms of HHT by the end of the 3rd decade; 2) laser was the modal treatment for HHT-related epistaxis; 3 ) 71 patients were screened for PAVMs $(57.7 \%)$; 4) computerized tomography (CT) was the modal screening tool (58 patients);

5) PAVMs were detected in 30 patients $(42.2 \%$ screened population); 6) Shortness of breath, and migraine more common in patients with PAVMs than those without, although 4/30 patients with PAVMs reported no symptoms or signs; 7) 18 patients received treatment for PAVMs; 8 ) Trans-catheter embolisation was the modal treatment for PAVMs (16 patients); 9) the data did not support the use of epistaxis severity scores to guide whether or not to offer screening.

The high prevalence of nose-bleeding as a primary symptom before the age of 30 in this series reflects both the setting of this study in an ENT referral centre and mirrors previous studies that suggest it is one of the earliest and most frequently occurring symptoms of the disease ${ }^{(20)}$. Indeed, a Norwegian study of $98 \mathrm{HHT}$ patients found $56 \%$ reported nosebleeds by 10 years of age ${ }^{(21)}$. However, it is of clinical significance that in this study some patients did not experience their first symptom until the 4 th or 5 th decade, with a minority reporting their first symptoms at even greater ages.

Whilst most patients experienced mild to moderate epistaxis in this study, 36.6\% experienced severe symptoms. Other studies have reported smaller proportions of severe cases using different methods of measuring epistaxis severity, including quality of life ${ }^{(20)}$ and intensity and frequency of nosebleeds and number of blood transfusions ${ }^{(21)}$. Our data from a major tertiary referral centre may be biased by a tendency of practitioners to refer HHT patients with more severe nosebleeds and manage mild and moderate cases in primary or secondary care. The reported worsening of epistaxis symptoms with age 


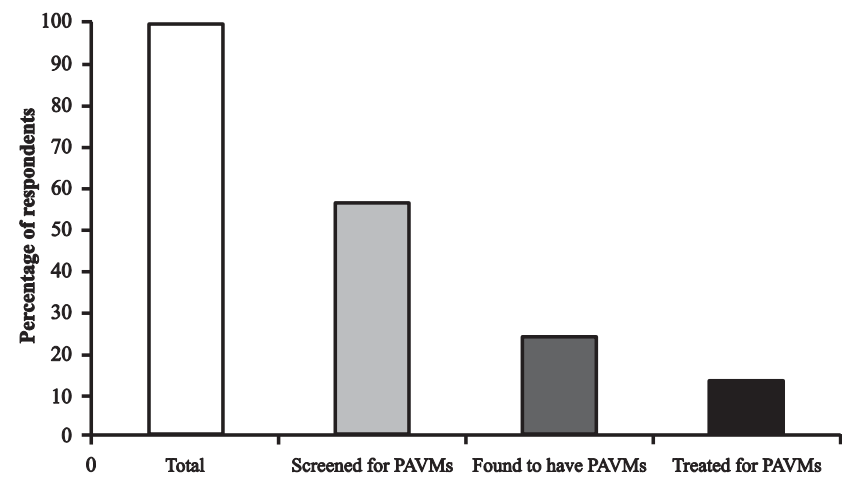

Figure 3. Outcome of screening procedures. Respondents $(n=123)$ were asked about investigations, results and treatment for pulmonary arteriovenous malformations (PAVMs).

amongst $86.2 \%$ of this study population echoes the findings of a number of published studies that suggest a trend towards progression of epistaxis with age ${ }^{(20,21)}$. Larger studies are required to confirm whether this truly reflects the natural history of the disease. It is known that manifestations of HHT are not seen at birth but develop and may sometimes exacerbate with age, with visceral (lung, gastrointestinal) malformations frequently occurring after the onset of nosebleeds ${ }^{(4,15)}$. The great variability in epistaxis severity in this study is well described in the literature and is currently speculated to be due to the interaction of multiple genetic factors in the pathogenesis of the disease ${ }^{(4)}$. Chronic nasal and gastrointestinal haemorrhage frequently produces iron-deficiency anaemia, which is likely to explain the large number of patients who reported the use of iron supplements.

Most patients were treated for epistaxis using laser, reflecting guidelines developed at the Royal National Throat Nose and Ear Hospital by author VJL ${ }^{(22)}$. Several patients reported the use of cautery and nasal packs to treat HHT-related epistaxis, which can be counterproductive ${ }^{(22)}$.

PAVMs have been reported in the literature as occasionally 'silent' or asymptomatic at the time of stroke or brain abscess ${ }^{(8)}$. Although only a small number of patients were asymptomatic in this study, it is important to note that patients without PAVMs were no more likely to be asymptomatic than those with PAVMs. This highlights the importance of screening regardless of symptoms. Symptoms of dyspnoea, chest pain, haemoptysis and migraine were all reported in patients with PAVMs in this study, and are known to be associated with PAVMs in major series assessing symptoms at initial presentations ${ }^{(9,14,15,23-30)}$. Although migraine and dyspnoea were reported significantly more amongst the PAVM group in this study, these symptoms are far from being useful as clinical predictors for PAVMs, as there was a high background reporting rate of these symptoms in the group without PAVMs. Despite no statistcal difference between the two groups, brain abscesses were only reported amongst the group with PAVMs, which may reflect both the sample size and a relatively low incidence of this complication overall. The limitations of recall bias, combined with an inability to assess symptoms pre-operatively in every patient made it difficult to assess true symptomatology in this retrospective study. Unfortunately it was not possible to assess clinical signs such as cyanosis, clubbing or vascular bruit using this study design, but these have also been associated with the presence of PAVMs ${ }^{(15,23)}$.

The number of patients yet to be screened for PAVMs (41\%) compared to guideline recommendations for $100 \%$ universal screening may show the difficulties of long-term follow-up in a 25-year cohort of patients, many of whom were diagnosed and treated for epistaxis before international guidelines were published in 2009.

The results for PAVM management reflect the fact that the major HHT centre in the UK (Hammersmith) has adopted thoracic $\mathrm{CT}$ as a first-line tool, weighing the additional radiation exposure against substantial time and resources required for a multi-step system that includes trans-thoracic contrast echocardiography (TTCE) ${ }^{(18,31,32)}$. The majority of treatable PAVMs (usually $>3 \mathrm{~mm}$ diameter) were managed with trans-catheter embolisation, reflecting the guidelines. The two surgical cases were due to very large PAVMs that were not referred for embolization because it was decided locally that they could not be treated by embolisation alone, although this does not reflect Hammersmith practice where even large PAVMs are treated by embolisation.

The recommendation to screen all patients for PAVMs regardless of the symptoms of HHT is strengthened by the finding that neither age of onset of HHT symptoms nor mean epistaxis 
severity score significantly differed between patients with PAVMs and those without. Moreover, the fact that mean epistaxis severity did not differ between the screened and unscreened populations makes it unlikely that patient selection has contributed to a lack of screening for PAVMs.

Although a thorough investigation of all screening procedures in HHT patients is beyond the scope of this study, it is important to note that pulmonary AVMs are not the sole visceral malformation that pose a risk to this patient group. The international guidelines also include a weak recommendation for routine screening for cerebral AVMs, although there was some dissent among authors due to a lack of evidence for effective treatment for this complication. Screening for hepatic AVMs is indicated only if there are abnormal liver enzymes and a clinical picture suggesting complications (high output heart failure, portal hypertension, jaundice). Screening for gastrointestinal AVMs is recommended to begin over 35 yrs old with measurement of haemoglobin and endoscopy for those whose anaemia is disproportionate to their epistaxis ${ }^{(17)}$

\section{Conclusion}

This study showed that in a large series of HHT patients, the majority received screening for PAVMs in line with current international guidelines. ENT surgeons are well placed to refer patients with epistaxis for screening, but numbers of screened patients have yet to reach target levels set by international guidelines. Whilst most patients had first symptoms of HHT at a young age, clinicians must be vigilant to the diagnosis throughout life in patients with any 2 of recurrent nosebleeds, multiple telangiectasia, visceral vascular malformations or positive family history ${ }^{(3)}$.

Clinicians should seek to advise all patients, former patients and their families with possible or confirmed HHT of the risks of PAVMs and the availability of screening and effective treatment. Guidelines recommend long-term follow-up in these patients, even for those with negative screens or untreated PAVMs. The findings of this study do not support the use of epistaxis severity scores or age of onset of epistaxis to inform whether or not to offer screening for PAVMs.

\section{Acknowledgements}

We gratefully acknowledge Vicki Holmes and the Medical Illustration Department at the RNTNE Hospital, London for help with figures, and extend our thanks to the patients and families involved in the study.

\section{Authorship contribution}

Conceived and designed the study: MMV, CLS, VJL

Collected and analyzed the data: MMV, VJL

Wrote the paper: MMV, CJS, VJL

\section{Conflict of interest}

None.

\section{References}

1. Kjeldsen AD, Vase P, Green A. Hereditary haemorrhagic telangiectasia: a populationbased study of prevalence and mortality in Danish patients. J Intern Med. 1999; 245: 31-39.

2. Begbie ME, Wallace GMF, Shovlin $C L$. Hereditary haemorrhagic telangiectasia (Osler-Weber-Rendu syndrome): a view from the 21st century Postgrad Med J. 2003; 79: 18-24.

3. Shovlin $C L$, Guttmacher $A E$, Buscarini $E$, et al. Diagnostic criteria for hereditary haemorrhagic telangiectasia (Rendu-Osler-Weber syndrome) Am J Med Genet. 2000; 91 : 66-67.

4. Govani FS, Shovlin CL. Hereditary haemorrhagic telangiectasia: a clinical and scientific review. European Journal of Human Genetics. 2009; 17: 860-871.

5. Rundles RW. Hemorrhagic telangiectasia with pulmonary artery aneurysm: case report. Am J Med Sci. 1945; 210: 76-81.

6. Smith JL, Lineback MI. Hereditary hemorrhagic telangiectasia, nine cases in one Negro family with special reference to hepatic lesions. Am J Med. 1954; 17: 41-49.

7. Roma'n G, Fisher M, Perl DP, et al. Neurological manifestations of hereditary hemorrhagic telangiectasia (Rendu-Osler-
Weber Disease): report of 2 cases and review of the literature. Ann Neurol. 1978; 4: 130-144.

8. Guttmacher AE, Marchuk DA, White RI: Hereditary hemorrhagic telangiectasia. New Engl J Med. 1995; 333: 918-924.

9. Cottin V, Plauchu H, Bayle J-Y, et al. Pulmonary arteriovenous malformations in patients with hereditary hemorrhagic telangiectasia. Am J Respir Crit Care Med. 2004; 169: 994-1000.

10. White R Jr, Lynch-Nylan A, Terry P, et al Pulmonary arteriovenous malformations: Techniques and long-term outcomes of embolotherapy. Radiology. 1988; 169: 663669.

11. Haitjema TJ, Overtoom TTC, Westermann CJJ, et al. Embolisation of pulmonary arteriovenous malformations: results and followup in 32 patients. Thorax. 1995; 50: 719-723.

12. Gupta P, Mordin C, Curtis J, et al. Pulmonary arteriovenous malformations: Effect of embolization on right-to-left shunt, hypoxaemia and exercise tolerance in 66 patients. Am J Roentgenol 2002; 179: 347-355.

13. Cottin V, Chinet T, Lavole' A et al. Pulmonary arteriovenous malformations in hereditary hemorrhagic telangiectasia patients: a series of 126 patients. Medicine (Baltimore). 2007; 86: 1-17.
14. Vase P, Holm M, Arendrup H. Pulmonary arteriovenous fistulas in hereditary hemorrhagic telangiectasia. Acta Med Scand. 1985; 218: 105-109.

15. Sluiter-Eringa $H$, Orie NGM, Sluiter $H J$. Pulmonary arteriovenous fistula. Diagnosis and prognosis in noncomplainant patients. Am Rev Respir Dis. 1969; 100: 177-188.

16. Tattersfield $A E$, Shovlin $C L$, Letarte, $M$ Hereditary haemorrhagic telangiectasia and pulmonary arteriovenous malformations: issues in clinical management and review of pathogenic mechanisms. Thorax. 1999; 54: 714-729

17. Faughnan ME, Palda VA, Garcia-Tsao G, et al. International Guidelines for the Diagnosis and Management of Hereditary Hemorrhagic Telangiectasia. J Med Genet. 2011; 48: 73-87.

18. Shovlin $C L$, Jackson JE, Bamford KB, et al. Primary determinants of ischaemic stroke/ brain abscess risks are independent of severity of pulmonary arteriovenous malformations in hereditary haemorrhagic telangiectasia. Thorax. 2008; 63: 259-266.

19. Hoag JB, Terry P, Mitchell S. An epistaxis severity score for hereditary hemorrhagic telangiectasia. Laryngoscope. 2010; 120: 838-843.

20. Aassar OS, Friedman CM, White RI. The natu- 
ral history of epistaxis in hereditary hemorrhagic telangiectasia. Laryngoscope. 1991; 101: 977-980

21. Dheyauldeen S, Abdelnoor M, BachmannHarildstad G. The natural history of epistaxis in patients with hereditary haemorrhagic telangiectasia in the Norwegian population: a cross-sectional study. Am J Rhinol Allergy. 2011; 25: 214-218.

22. Lund VJ, Howard DJ. A treatment algorithm for the management of epistaxis in hereditary haemorrhagic telangiectasia. American Journal of Rhinology 1999; 13: 319-322.

23. Dines DE, Seward JB, Bernatz PE. Pulmonary arteriovenous fistulas. Mayo Clin Proc. 1983; 58: 176-181.

24. Yater WM, Finnegan J, Giffin HM. Pulmonary arteriovenous fistula (varix). JAMA. 1949; 141: 581-589.

25. Shumacker HB, Waldhausen JA. Pulmonary arteriovenous fistulas in children. Ann Surg. 1963; 158: 713-720

26. Terry PB, White RI, Barth $\mathrm{KH}$, et al. Pulmonary arteriovenous malformations: physiologic observations and results of balloon embolisation. N Engl J Med. 1983; 308: 1197-1200.

27. Pennington DW, Gold WM, Gordon RL et al. Treatment of pulmonary arteriovenous malformations by therapeutic embolization. Am Rev Respir Dis. 1992; 145: 1047-1051.

28. Puskas JD, Allen MS, Moncure AC, et al. Pulmonary arteriovenous malformations: therapeutic options. Ann Thorac Surg. 1993; 56: 253258.

29. Dutton JAE, Jackson JE, Hughes JMB, et al Pulmonary arteriovenous malformations: results of treatment with coil embolisation in 53 patients. AJR. 1995; 165: 1119-1125.

30. Haitjema TJ, Overtoom TTC, Westermann CJ, et al. Embolisation of pulmonary arteriovenous malformations: results and followup in 32 patients. Thorax. 1995; 50: 719-723.

31. Shovlin CL. Hereditary haemorrhagic telangiectasia: pathophysiology, diagnosis and treatment. Blood Reviews 2010; 6: 203-219.

32. Shovlin $C L$, Wilmshurst $P$, Jackson JE Pulmonary arteriovenous malformations and other pulmonary aspects of hereditary haemorrhagic telangiectasia. ERM 2012, in press.

Professor Valerie J Lund

Professorial Unit,

Royal National Throat Nose and Ear

Hospital

330 Gray's Inn Road London

London

United Kingdom

Tel: +44-207915 1497

Fax: +44-207 8339480

E-mail: v.lund@ucl.ac.uk

\section{ADVERTISEMENT}

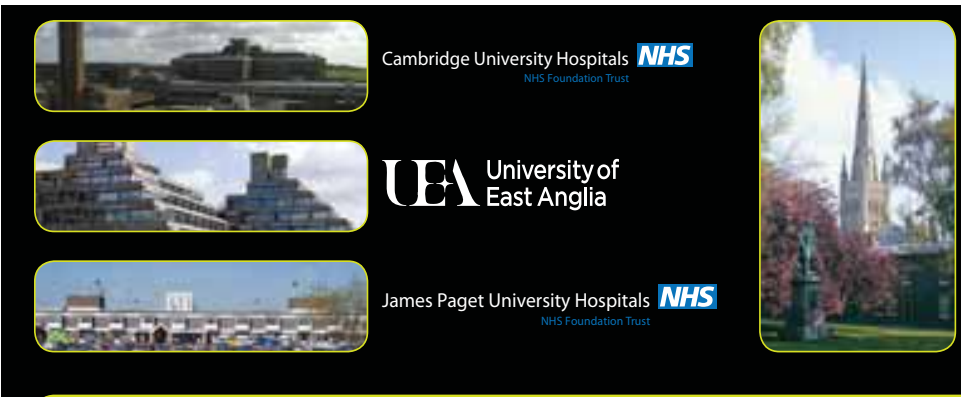

\section{2nd East Anglian Course on Management of Sinonasal Disease in the 21st Century}

Abstract

The course will comprise of hands on cadaveric dissection, live surgery demonstration (including image guided sinus surgery, balloon sinuplasty and sinus lavage in the outpatient clinic), lectures and panel discussions on controversies and emerging therapies in the management of sinonasal disease. The emphasis will be on chronic rhinosinusitis exploring current strategies regarding medical and The emphasis will be on chronic rhinosinusits, exploning curent strategies regarding medical and includes a course dinner and transportation between sites (Cambridge/Norwich/Great Yarmouth). Course organisers: Mr Carl Philpott / Mr David McKieman

Local faculty: Dr Andrew Wilson (Senior Lecturer UEA/Honorary Consultant Respiratory Physician Norfolk \& Norwich University Hospital

Dr Paddy Wilson (Consultant Radiologist, Norfolk \& Norwich University Hospital) Dr Silke Schelenz (Senior Lecturer UEA/Honorary Consultant Microbiologist, Norfolk \& Norwich University Hospital

Visiting faculty: Dr Amin Javer (Director of St Paul's Sinus Centre, Vancouver, Canada) Mr Sean Carrie (Consultant ENT Surgeon, Freeman Hospital, Newcastle) Miss Claire Hopkins (Consultant ENT Surgeon, Guys \& Thomas Hospital, London) Mr Anshul Sama (Consultant ENT Surgeon, Queens Medical Centre, Nottingham) Mr Sankalp Sunkaraneni (Rhinology Fellow, St Paul's Sinus Centre, Vancouver)

Date: $\quad$ 5-7th December 2012

Venue: $\quad$ Addenbrookes Hospital (Cambridge) / UEA (Norwich) / James Paget University Hospital (Great Yarmouth)

Course Fee: $\quad E 750$

Accreditation: $\quad$ ENT UK / RCSEng

Delegates: $\quad 20$; aimed at higher surgical trainees, consultants and non-consultant career grades

Sponsored by

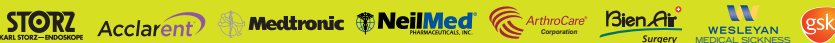

